\title{
Evolución de las poblaciones piscícolas del río Manzanares aguas abajo del embalse de El Pardo
}

\author{
M. Morillo González del Tánago, A. Giménez Miró \& D. García de Jalón Lastra \\ Lab. Hidrobiología, E.T.S.I. Montes, U.P.M., Avda. Ramiro de Maeztu s/n, 28040, Madrid
}

\begin{abstract}
RESUMEN
Se han estudiado los cambios en la comunidad de peces del rio Manzanares por debajo del embalse del Pardo. La comunidad de peces antes del embalse estaba constituida principalmente por ciprínidos autóctonos. Durante el periodo 1989-1997, las especies no nativas constituían el $80 \%$ de la comunidad. El análisis del régimen de flujos antes y después de la construcción del embalse mostraba grandes cambios en la magnitud, frecuencia y periodicidad del flujo. Esto ha provocado cambios dramáticos en la geomorfología del río y un gran crecimiento de la vegetación riparia aguas abajo, que ha favorecido a las especies exóticas, actualmente en expansión.
\end{abstract}

Palabras clave: ictiofauna, regulación de caudales, especies exóticas, cambios del habitat, desaparición de especies autóctonas

\begin{abstract}
Changes in the fish community of the River Manzanares, downstream of El Pardo Reservoir (Madrid, Spain) have been studied. The pre-impoundment fish community was mainly constituted by autochthonous cyprinids. During the period 19891997, non-native species comprised roughly $80 \%$ of the community. The analysis of flow regimes before and after the construction of the dam showed drastic changes in the magnitude, frequency and periodicity of flow. This has lead to dramatic changes in the geomorphology of the river, and to the development of luxuriant growth of riparian vegetation downstream. This has favoured the expansion of exotic species.
\end{abstract}

Keywords: ichthyofauna, impoundment, exotic fish, habitat changes, lost native species.

\section{INTRODUCCIÓN}

La construcción de presas es, junto con la destrucción del hábitat, la introducción de especies exóticas y la contaminación de las aguas, uno de los factores que más afectan a las poblaciones piscícolas autóctonas (Granado-Lorencio, 1995; Elvira, 1996). En concreto, en España, donde la construcción de presas y embalses se ha visto promovida y justificada por el supuesto desequilibrio hídrico existente, el problema es especialmente grave. Hoy en día todos los grandes río españoles están regulados por embalses, y lo mismo sucede con la mayoría de los ríos medianos (García de Jalón, 1987; García de Jalón et al., 1992). Los efectos de este tipo de estructuras sobre las comunidades piscícolas fluviales son notables dado que rompen el continuo fluvial (Vannote et al., 1980). A corto plazo interrumpen las rutas migratorias, con la consiguiente desaparición de las especies (Horváth, 1998). A largo plazo, las presas y embalses provocan fuertes cambios en el hábitat y la desaparición de las comunidades ícticas originales, que son sustituidas por especies exóticas (Martinez et al., 1994).

\section{El río Manzanares}

La cuenca del Manzanares constituye uno de los espacios de la Península Ibérica más estudiados, 
disponiéndose de gran número de trabajos tanto teóricos como aplicados. En sus aguas se realizó el primer estudio sobre ecología fluvial de un río español (Velaz de Medrano y Ugarte, 1933). Dentro de los estudios realizados posteriormente sobre la ictiofauna del río Manzanares cabe destacar los trabajos de Doadrio (1984), Montes et al. (1987), y Casado et al. (1987). Este río como muchos otros ha sido objeto de introducciones con mayor o menor éxito (lucio, black-bass, pez gato y pez sol entre otras) cuyos efectos pretendemos estudiar. Asimismo se han analizado los efectos de la regulación del régimen de caudales tras la construcción de la presa de El Pardo, los cambios morfológicos sufridos por el cauce y sus efectos sobre las poblaciones piscícolas residentes.

\section{ÁREA DE ESTUDIO}

El río Manzanares es el tercer afluente por la margen derecha del Jarama (Cuenca del Tajo). Nace a unos 2.160 m.s.n.m. en la vertiente meridional de la Sierra de Guadarrama. A lo largo de su recorrido por la provincia de Madrid está regulado por dos embalses, el de Santillana, situado en el término municipal de Manzanares el Real, destinado al abastecimiento de agua, y el embalse de El Pardo, en la población homónima y cuya función es la regulación del Manzanares a su paso por el área metropolitana de Madrid.

La presa de El Pardo fue terminada en el año 1970. El dique es de tierra, de $35 \mathrm{~m}$ de altura y $830 \mathrm{~m}$ de longitud en coronación. El embalse tiene una capacidad de $45 \mathrm{hm}^{3}$, cubre una superficie de 550 ha y está dotado de un aliviadero de compuertas con capacidad para desaguar $750 \mathrm{~m}^{3} / \mathrm{s}$.

El área de estudio se sitúa aguas abajo de la presa de El Pardo a unos 600 m.s.n.m. sobre un sustrato de depósitos aluviales del Cuaternario. Corresponde al tramo bajo (potamon), con aguas relativamente lentas, un sustrato de arenas y cantos, y una vegetación de ribera constituida por saucedas salvifolias oligótrofas con S. salviifolia, $S$. atrocinerea y $F$. angustifolia (Varela et al., 1996).
El muestreo piscícola se realizó aguas abajo de la estación de aforos de El Pardo (E.A. n ${ }^{\circ} 187$ ), a una distancia de $825 \mathrm{~m}$ del embalse. Las características del punto de muestreo se resumen en la Tabla 1.

\section{MATERIAL Y MÉTODOS}

\section{Poblaciones piscícolas}

El muestreo piscícola fue realizado en seis campañas en la estación de otoño (meses de OctubreNoviembre) a lo largo de los años 1989-91 y 1994-96. En 1997, se realizó un muestreo adicional en verano, mientras que en otoño no fue posible debido al elevado caudal circulante.

Como método de captura se seleccionó la pesca eléctrica, siendo el método de muestreo de "extracción sin reemplazo" o pasadas sucesivas sin devolución (método de De Lury, 1947) por su sencillez y el menor trabajo y tiempo que conllevan sus operaciones de muestreo con relación a otros métodos. Se dieron un mínimo de dos pasadas, más una tercera adicional cuando se hizo patente que las sucesivas capturas seguían una pauta poco adecuada.

El tramo fue cerrado con una red de agalla aguas abajo, aprovechando la existencia del azud de la estación de aforos como cota superior, que impide la entrada o salida de peces del área.

Las pasadas fueron cronometradas con objeto de que su duración fuese similar y que el

Tabla 1. Características del área de muestreo piscícola. Characteristics of the sampling area.

\begin{tabular}{lc}
\hline Coordenadas U.T.M. & 30TUK331852 \\
\hline Área muestreada $\left(\mathrm{m}^{2}\right)$ & $550-620$ \\
Anchura media $(\mathrm{m})$ & $14-30$ \\
Profundidad máxima $(\mathrm{cm})$ & 69 \\
Longitud de la sección $(\mathrm{m})$ & $30-60$ \\
Estructura del lecho & cantos $<$ arena $<$ gravilla \\
Tipo de tramo & rápido \\
\hline
\end{tabular}


esfuerzo de pesca y probabilidad de captura se mantuviesen constantes entre pasadas, según establece Morán (1951).

Los peces capturados fueron estabulados en barreños de $30 \mathrm{~L}$ de capacidad con agua fresca. Una vez finalizada la pasada y cuando los peces se apreciaron más calmados, se procedió a la toma de datos de cada individuo: longitud hasta la horquilladura y peso. Para la determinación de la edad se tomaron escamas de una submuestra de los individuos capturados, seleccionándose para ello la región comprendida entre la línea lateral y la aleta dorsal (Granado-Lorencio, 1996).

Cuando fue posible determinarlo a simple vista también se anotó el sexo de cada uno de los ejemplares.

\section{Caracterización de las poblaciones piscícolas}

La caracterización de la comunidad íctica se realizó en base a su composición faunística, determinando para ello su riqueza y diversidad de Shannon-Weaver en cada uno de los muestreos, las especies dominantes y subdominantes. Asimismo se calcularon los valores de densidad y biomasa.

Por otra parte, para la caracterización de las poblaciones piscícolas se determinó la estructura en tamaños de la población, longitudes y pesos medios de cada clase de edad y los valores de densidad y biomasa instantáneas de cada una de los muestreos realizados. La metodología seguida para el cálculo de estos parámetros se expone a continuación.

\section{Determinación de la edad. Estructura de la población}

Para la determinación de la edad se analizó en el laboratorio la submuestra de escamas recogidas, que fueron "leídas" con ayuda de una lupa binocular de aumento variable entre 6x y 50x. E1 resto de los individuos fueron asignados por extrapolación, con la ayuda del gráfico de frecuencias de longitudes - Método de Petersen (1896 en De Vries \& Fry, 1996).

\section{Longitud y pesos medios. Relaciones de longitud/peso}

Una vez determinadas las edades, se calcularon las longitudes y pesos medios correspondientes a cada clase de edad y los correspondientes errores estándar con un nivel de confianza del 95\%, con ayuda de los cuales determinamos asimismo las relaciones longitud-peso características de cada especie a lo largo de las campañas de muestreo. Para ello se calculó el factor de condición $\mathrm{K}(\mathrm{K}=$ peso $\mathrm{x}$ $100 /$ long $^{3}$ ). Por otra parte, la relación longitudpeso, viene dada por la siguiente expresión: peso $=\mathrm{a} \times$ long $^{\mathrm{b}}$ (Anderson \& Neuman, 1996).

\section{Estructura cualitativa. Densidad y biomasa}

Para realizar la estimación del número total de individuos presentes en el tramo se aplicó el método de Máxima Verosimilitud de Morán \& Zippin (1958) dado su mayor rigor estadístico (LobónCerviá, 1991). En los casos en los que tan solo se pudieron realizar dos pasadas se utilizó el método de máxima verosimilitud ponderada de Carle \& Strub (1978) por su mayor robustez estadística.

\section{Análisis de caudales}

Las comunidades fluviales pueden estar reguladas por factores de tipo biótico o abiótico (Peckarski, 1983). En el caso que nos ocupa, el punto de muestreo piscícola se encuentra aguas abajo de un embalse, lo que supone un fuerte estrés ambiental. Por ello, y ante las fuertes oscilaciones observadas en las poblaciones que componen la comunidad íctica, hemos analizado los caudales circulantes antes y después de la construcción de la presa como uno de los posibles factores reguladores de la comunidad.

Se han utilizado los datos de caudales correspondientes a la estación de aforos número 70 de la Confederación Hidrográfica del Tajo, situada en el Parque Sindical (coordenadas: 3-45-32 O, 40-27-45 N). Esta estación ha sido seleccionada pese a existir otra más próxima, localizada en el tramo de estudio (E.A. $n^{\circ}$ 187, El Pardo), dado 
que tiene registros de caudales anteriores a la construcción de la presa de El Pardo. La comparación de los datos de las dos estaciones de aforos no dio como resultado diferencias significativas para $\mathrm{p}<0.01$. Para definir el régimen de caudales anterior a la construcción de la presa hemos tomado la serie de datos de octubre de 1940 a septiembre de 1969. El régimen actual queda definido por los datos de aforos desde octubre de 1969 a septiembre de 1994, último año hidrológico del que hay datos disponibles. De este modo pretendemos estudiar la evolución del régimen de caudales como consecuencia del efecto regulador del embalse. Para ello se calcularon los caudales medios mensuales en ambos periodos (antes y después del embalse) y las medias de los máximos. A partir de éstos se determinó el módulo anual y el coeficiente de variación. También se determinaron las frecuencias absolutas y acumuladas de los caudales medios diarios de ambos periodos (antes y después de la construcción del embalse de El Pardo).

\section{Estudio morfológico}

La regulación artificial de caudales tiene profundos efectos sobre la fauna piscícola, al modificar el hábitat fluvial aguas abajo de un embalse (García de Jalón, 1990), cambios que pueden ser perjudiciales para las comunidades piscícolas residentes. Por lo tanto, para completar el estudio se analizaron los cambios morfológicos sufridos por el tramo tras la construcción de la presa de El Pardo. Se realizaron un total de 30 transectos o secciones transversales con el objeto de representar todas aquellas formas fluviales significativas o distintas. En cada uno se anotaron, en celdas equidistantes, la distancia a la orilla, profundidad, granulometría del lecho (basada en la clasificación de la American Geophysical Union), la cobertura de sombra, vegetación acuática, y velocidad de la corriente. También se tomaron datos referentes a la forma de las orillas, los taludes laterales y la pendiente de la lámina de agua a lo largo del tramo. Asimismo, para la determinación de la capacidad de refugio o de cobertura para las poblaciones piscícolas se tomó nota de la presencia de todos aquellos objetos naturales o artificiales que pudieran suministrar una protección frente a la corriente y la insolación, o que sirvieran de refugio ante la proximidad de un peligro.

El análisis se completó mediante la comparación de fotografías aéreas de los años 1968 (E: 1/4500), 1972 (E: 1/7500) y 1991 (E: 1/6500). Esto nos ha permitido evaluar la transformación morfológica del río en este tramo tras la construcción de la presa de El Pardo.

Como herramienta de trabajo a la hora de obtener representaciones gráficas así como cuantificaciones de los cambios acaecidos hemos utilizado el Sistema de Información Geográfico IDRISI, desarrollado por la Universidad de Clark, Massachussets. Este programa permite suponer fotografías aéreas de diferentes escalas, y por tanto, analizar la variación de cada punto a lo largo del tiempo.

\section{RESULTADOS}

\section{Composición de la comunidad piscícola}

Se expone a continuación la relación de especies capturadas en el tramo de estudio:

\section{Fam. CYPRINIDAE}

Barbus bocagei Steindachner, 1865

Cyprinus carpio Linnaeus, 1758

Chondrostom polylepis Steindachner, 1865

Gobio gobio (Linnaeus, 1758)

(barbo común) (carpa común)

(boga de río)

(gobio)

Fam. POECILIIDAE

Gambusia holbrooki Girard, 1859

(gambusia)

Fam. CENTRARCHIDAE

Micropterus salmoides (Lacépède, 1802) （black-bass)

Lepomis gibbosus (Linnaeus, 1802)

(pez sol)

Fam. ESOCIDAE

Esox lucius Linnaeus, 1758

(lucio)

\section{Fam. ICTALURIDAE}

Ameiurus melas (Rafinesque, 1820)

(pez gato) 
La comunidad piscícola en este tramo está compuesta por diez especies pertenecientes a cinco familias, cuatro de ellas exóticas en nuestras aguas (Centrarchidae, Esocidae, Ictaluridae y Poeciliidae) lo que supone el $80 \%$ del total. De hecho, tan solo dos especies son autóctonas en nuestras aguas: el barbo común y la boga de río.

La riqueza faunística oscila entre un mínimo de 6 y un máximo de 8 taxa (Tabla 2). La diversidad, en general es muy baja $(\mathrm{H}<1$ bit), indicio de una comunidad degradada (Fausch et al., 1990).

\section{Evolución de la comunidad piscícola: composición, densidad y biomasa}

En líneas generales se observan fuertes cambios tanto en la densidad como en la composición, con dominancia (entendida como la especie de mayor densidad y biomasa) alternada a lo largo de los años muestreados (Tabla 2). Así en ambos periodos (1989-91, 1994-96), la densidad aumenta, pero mientras de 1989-90 la especie dominante es el barbo, en 1991 la población de gobio duplica la del barbo $\left(0.58 \mathrm{ind} / \mathrm{m}^{2}\right.$ frente a $\left.0.26 \mathrm{ind} / \mathrm{m}^{2}\right)$ (ver Tabla 3 ).

En el siguiente periodo (1994-97) comienza dominando el barbo (1994), dando paso al pez sol, especie dominante durante el año 95, que a su vez resulta desplazada por el black-bass que se convierte en la especie dominante de la comunidad, con una densidad casi diez veces superior a cualquiera de las restantes especies en 1996 (ver Tabla 3).

La densidad de la comunidad íctica a lo largo de los distintos muestreos realizados oscila entre 0.12 y $1.64 \mathrm{ind} / \mathrm{m}^{2}$. Estos valores son ele-

Tabla 2. Evolución de la comunidad piscícola en términos de $\mathrm{n}^{\mathrm{o}}$ individuos, $\mathrm{n}^{\mathrm{o}}$ especies (riqueza faunística), biomasa ( $\mathrm{g} \mathrm{m}^{-2}$ ), densidad (ind $\mathrm{m}^{-2}$ ), diversidad (bits) y especies dominantes y subdominantes (v: verano). Changes in the fish community, expressed in number of individuals, number of species (faunistic richness), biomass $\left(\mathrm{g} \mathrm{m}^{-2}\right)$, density (ind $\left.\mathrm{m}^{-2}\right)$, diversity (bits) and dominant and subdominant species ( $v$ : summer).

\begin{tabular}{|c|c|c|c|c|c|c|c|}
\hline Muestreo & $\mathbf{N}^{0}$ spp. & $\mathbf{N}^{0}$ ind. & $\mathbf{B}_{\mathrm{T}}$ & $\mathbf{D}_{\mathrm{T}}$ & Diversidad & Sp. dominante & Sp. subdominante \\
\hline 1989 & 6 & 279 & 79.96 & 0.66 & 1.74 & barbo & p. sol \\
\hline 1990 & 7 & 112 & 99.44 & 1.01 & & barbo & p. sol \\
\hline 1991 & 6 & 444 & 54.69 & 1.64 & 0.54 & gobio/p.sol & barbo \\
\hline 1994 & 6 & 103 & 17.92 & 0.12 & 0.50 & barbo & carpa \\
\hline 1995 & 8 & 563 & 59.37 & 0.76 & 0.55 & p. sol & barbo \\
\hline 1996 & 7 & 218 & 16.35 & 1.39 & 0.52 & b-bass & p.sol \\
\hline $1997 v$ & 7 & 171 & 56.92 & 1.15 & 0.94 & p. sol & carpín \\
\hline
\end{tabular}

Tabla 3. Evolución de la densidad (ind $\mathrm{m}^{-2}$ ) de las poblaciones piscícolas del río Manzanares aguas abajo del embalse de El Pardo (v: verano; -: no se ha capturado ningún ejemplar). Changes in density (ind $\mathrm{m}^{-2}$ ) of fish populations in the River Manzanares downstream of El Pardo reservoir (v: summer, -: no individuals were caught).

\begin{tabular}{llllllll}
\hline & $\mathbf{1 9 8 9}$ & $\mathbf{1 9 9 0}$ & $\mathbf{1 9 9 1}$ & $\mathbf{1 9 9 4}$ & $\mathbf{1 9 9 5}$ & $\mathbf{1 9 9 6}$ & $\mathbf{1 9 9 7 v}$ \\
\hline Barbo & 0.41 & 0.88 & 0.26 & 0.04 & 0.22 & 0.11 & 0.06 \\
Gobio & 0.05 & 0.11 & 0.58 & 0.01 & 0.00 & - & - \\
Lucio & 0.00 & - & - & - & 0.00 & - & - \\
P. sol & 0.13 & 0.01 & 0.56 & 0.00 & 0.29 & 0.22 & 0.90 \\
B. bass & - & - & - & 0.01 & 0.01 & 0.98 & 0.01 \\
P. gato & - & - & - & 0.02 & 0.15 & 0.05 & 0.00 \\
Gambusia & 0.06 & 0.01 & 0.24 & - & 0.08 & - & 0.05 \\
Carpa & 0.01 & - & - & 0.03 & - & 0.003 & 0.02 \\
Carpín & - & - & - & 0.00 & - & 0.002 & 0.10 \\
Boga & - & - & - & - & 0.002 & - & - \\
TOTAL & 0.66 & 1.01 & 1.64 & 0.12 & 0.76 & 1.39 & 1.15 \\
\hline
\end{tabular}


vados si los comparamos con los obtenidos por Casado et al. (1987) que tan sólo alcanzaron 0.07 ind $/ \mathrm{m}^{2}$ en la campaña de otoño y 0.51 ind $/ \mathrm{m}^{2}$ en primavera. No obstante, resultan bastante bajos en comparación con estudios realizados en otros ríos ibéricos. Así, Lobón-Cerviá \& Penzack (1984) obtuvieron, para el río Jarama, valores de densidad que oscilaban entre 1.3 y $8.5 \mathrm{ind} / \mathrm{m}^{2}$.

El comportamiento de la biomasa por otra parte, corresponde al de la población del barbo que constituye, por sí solo, más del $90 \%$ del total en casi todos los años muestreados. Se observan pautas semejantes a la densidad: la media de todos los años $\left(53.28 \mathrm{~g} / \mathrm{m}^{2}\right)$ es tres veces superior a la estimada por Casado et al. (1987) $\left(16.69 \mathrm{~g} / \mathrm{m}^{2}\right)$ para la misma época de muestreo (octubre 1987). Los valores obtenidos son sumamente elevados en comparación con otros ríos naturales de la Península cuya biomasa media oscila entre 14 y $18 \mathrm{~g} / \mathrm{m}^{2}$ (García de Jalón et al., 1996). No obstante, resultan perfectamente lógicos teniendo en cuenta que estamos ante un río regulado cuyo cauce está estabilizado.

Comparando los dos periodos de muestreo anteriormente mencionados no se observan diferencias significativas $(p>0.05)$ en cuanto a los valores de densidad estimados. Sin embargo, se observa una fuerte reducción de la biomasa $(p<0.05)$ cuyos valores medios pasan de 75.36 $\mathrm{g} / \mathrm{m}^{2}$ a $31.21 \mathrm{~g} / \mathrm{m}^{2}$, menos de la mitad.
Por especies, podemos observar los siguientes resultados:

a) B. bocagei Steindachner (barbo común).

Es la especie más representativa del tramo estudiado, apareciendo en todos los muestreos realizados. Como ya se comentó previamente, constituye por sí sola más del $90 \%$ del valor total de la biomasa de la comunidad excepto en los dos últimos años de muestreo, en los que la población se ve fuertemente mermada, al tiempo que otras especies de gran tamaño como el black-bass, la carpa y el carpín cobran cierta importancia (ver Tablas 3 y 4).

La población sufre fuertes fluctuaciones (expansiones y recesiones) de carácter periódico: de 1989 a 1990 duplica su población; de 1990 a 1991 la población se reduce a un tercio, alcanzando en 1994 su densidad mínima. Asimismo, en el siguiente periodo, entre 1994 y 1995, la población se quintuplica y en 1996 se reduce a la mitad (Tabla 3 ).

b) G. gobio L. (gobio).

La población de gobio, al igual que sucede con el barbo, sufre oscilaciones periódicas. En el primer periodo de estudio se puede observar una clara expansión, con valores de densidad que pasan de $0.05 \mathrm{ind} / \mathrm{m}^{2}$ alcanzados en 1989 , hasta los 0.58 $\mathrm{ind} / \mathrm{m}^{2}$ de 1991 (más de diez veces mayor), año en el cual se convierte en la especie dominante de la comunidad (ver Tablas 2 a 4).

Tabla 4. Evolución de la biomasa $\left(\mathrm{g} \mathrm{m}^{-2}\right)$ de las poblaciones piscícolas del río Manzanares aguas abajo del embalse de El Pardo (v: verano; -: no se ha capturado ningún ejemplar). Changes in biomass $\left(\mathrm{g} \mathrm{m}^{-2}\right)$ in the River Manzanares downstream of El Pardo reservoir (v: summer, -: no individuals were caught)

\begin{tabular}{lccccccc}
\hline & $\mathbf{1 9 8 9}$ & $\mathbf{1 9 9 0}$ & $\mathbf{1 9 9 1}$ & $\mathbf{1 9 9 4}$ & $\mathbf{1 9 9 5}$ & $\mathbf{1 9 9 6}$ & $\mathbf{1 9 9 7 v}$ \\
\hline Barbo & 69.71 & 97.97 & 50.13 & 17.18 & 55.54 & 12.43 & 23.77 \\
Gobio & 0.35 & 1.38 & 3.34 & 0.12 & 0.01 & - & - \\
Lucio & 0.33 & - & - & - & 0.38 & - & - \\
P. sol & 0.00 & 0.09 & 1.01 & 0.05 & 1.15 & 1.96 & 12.02 \\
B. bass & - & - & - & 0.18 & 0.84 & 0.66 & 4.71 \\
P. gato & - & - & - & 0.05 & 1.31 & 0.66 & 0.02 \\
Gambusia & 0.03 & 0.00 & 0.21 & - & 0.01 & - & 0.01 \\
Carpa & 1.49 & - & - & 0.36 & - & 0.61 & 11.86 \\
Carpín & - & - & - & 0.03 & - & 0.03 & 4.53 \\
Boga & - & - & - & - & 0.18 & - & - \\
TOTAL & 71.96 & 99.44 & 54.69 & 17.92 & 59.37 & 16.35 & 56.92 \\
\hline
\end{tabular}


En el segundo periodo de muestreo (1994 a 1996), sufre un receso hasta prácticamente "desaparecer". De hecho ni en otoño de 1996 ni en verano de 1997 se capturó ningún individuo de esta especie (Tabla 3 ).

Es una especie relativamente exigente en cuanto a la calidad de las aguas, que requiere un sustrato limpio, arenoso o de grava (sin limos), con una corriente moderada (Doadrio et al., 1990).

\section{c) L. gibbosus L. (pez sol).}

La perca sol o pez sol es otra especie alóctona cuya población sigue un patrón de oscilaciones anuales semejante al anterior: la población crece en 1989, sufre un fuerte receso al año siguiente (1990) y vuelve a expandirse en 1991 (ver Tabla 3).

Durante el periodo 1992-1994, se produjo una fuerte sequía en todo el país y a consecuencia de ello en 1994 alcanza el mínimo valor registrado $\left(0.002 \mathrm{ind} / \mathrm{m}^{2}\right)$. No obstante, a partir de entonces la población comienza a expandirse de nuevo y en el verano de 1997 alcanza la mayor densidad muestreada, $0.901 \mathrm{ind} / \mathrm{m}^{2}$ (el 78\% del total de la comunidad piscícola). d) M. salmoides Lacépède (black-bass).

Se trata de otra especie exótica de la misma familia que el pez sol, cuya presencia no es registrada hasta el segundo periodo de muestreo (año 1994). Mantiene una población relativamente estable, si bien en 1996 se convierte en la especie dominante de la comunidad, aunque todos los individuos capturados en este muestreo eran alevines del año (0+). En 1997 (verano) tan sólo se capturaron un alevín y un adulto de 6 años. Este último es el individuo más viejo datado de esta especie, y es probable que provenga del embalse cuando este vierte por coronación.

e) A. melas Rafinesque (pez gato).

Esta especie, también exótica en nuestras aguas, aparece en el año 1991 (1 ind). En los años 94-95 la población se mantiene más o menos estable, disminuyendo durante el año 1996 (Tabla 3).

f) G. holbrooki Girard (gambusia).

Esta especie tiene poblaciones relativamente escasas que no alcanzan siquiera los $0.1 \mathrm{ind} / \mathrm{m}^{2}$ en la mayor parte de los años muestreados (Tabla 3).

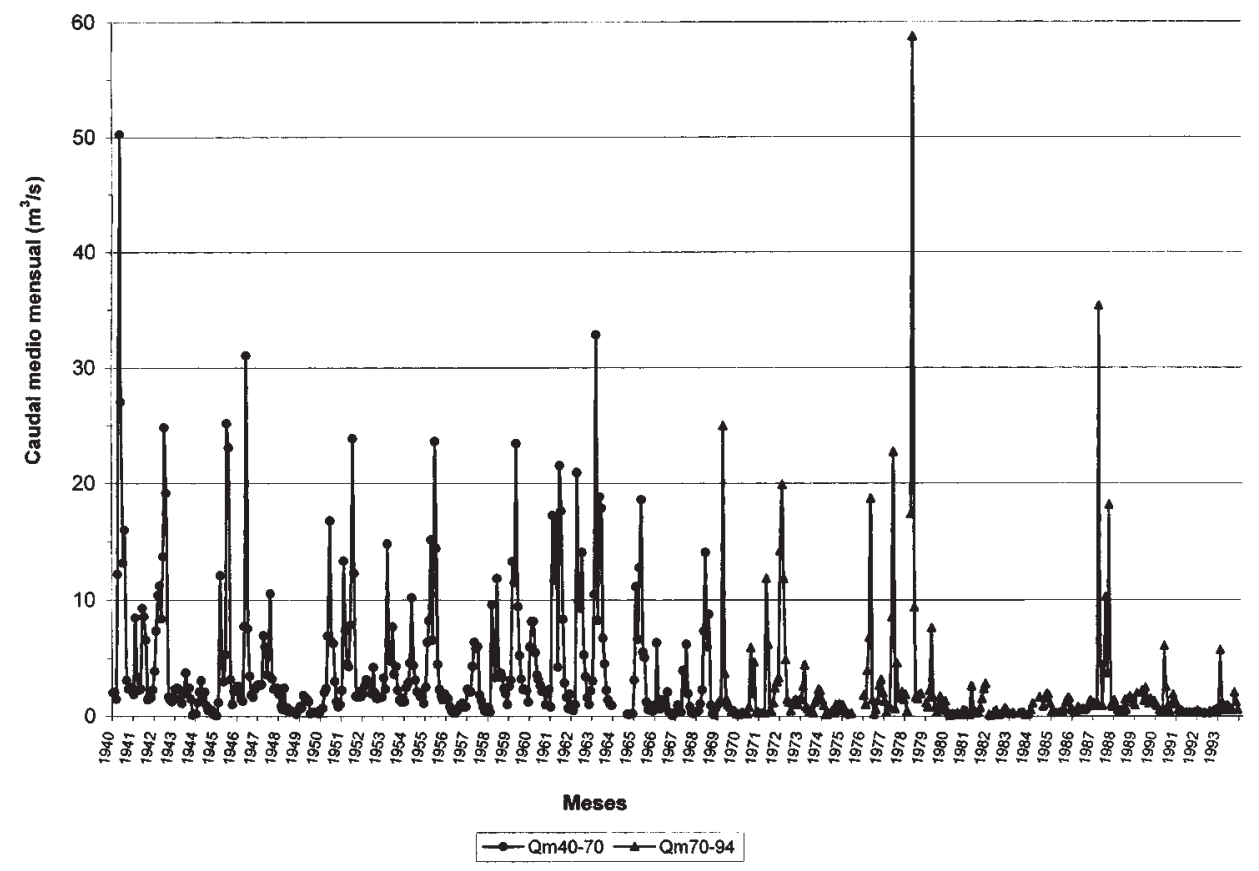

Figura 1. Caudales medios mensuales a lo largo del periodo 1940-1994. Monthly average discharges from 1940 to 1994. 
Tan solo en el año1991 supera este nivel, alcanzando $0.24 \mathrm{ind} / \mathrm{m}^{2}$, aunque esto sólo supone $15 \%$ escaso del total de la comunidad. En el segundo periodo de muestreo (1994-1997) su aparición es esporádica (Tabla 3 ).

Para el resto de las especies muestreadas $C$. carpio, C. auratus, Ch. polylepis y E. lucius, dado el escaso número de individuos capturados, tan sólo podemos mencionar su presencia.

\section{DISCUSIÓN}

Hemos visto en los resultados que la comunidad piscícola del tramo del río Manzanares regulado por el embalse se caracteriza por una gran inestabilidad tanto en su estructura como en su composición. Encontramos dos factores fundamentales a la hora de explicar la evolución de la comunidad piscícola: por una parte, la alteración del hábitat derivada de la regulación de caudales y, por otra, la introducción de especies exóticas.

\section{El régimen de caudales y el hábitat físico}

El análisis comparativo de los caudales circulantes antes y después de la construcción de la presa dio como resultado una homogeneización del régimen tras la construcción del embalse de El Pardo (ver Fig. 1), lo que nos lleva a pensar en

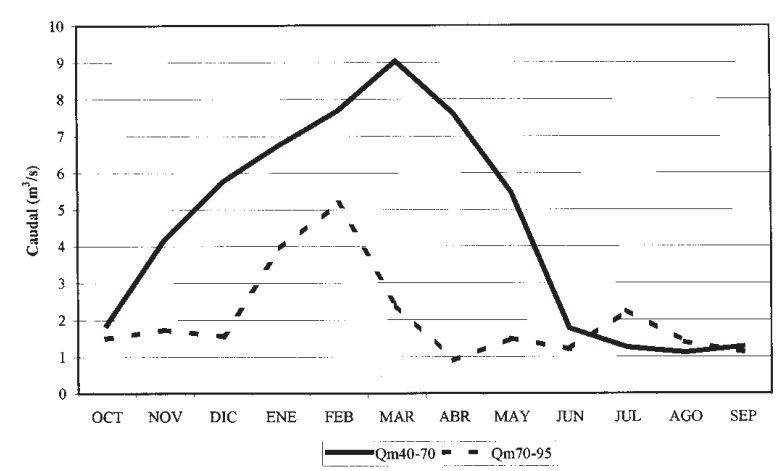

Figura 2. Comparación del régimen de caudales antes y después de la construcción de la presa de El Pardo. Comparison of annual flows before and after construction of the reservoir. este hecho como un importante factor regulador de la comunidad piscícola ya que las variaciones estacionales del caudal son cruciales en los ciclos biológicos de muchas especies fluviales (Welcomme, 1985). En efecto, las asociaciones ícticas de la Península Ibérica en general tienen un comportamiento vital que se corresponde con la mejor adaptación posible a un ambiente fluctuante y que representa variaciones irregulares a lo largo del tiempo (García de Jalón, 1990; Granado-Lorencio, 1995). Por lo tanto la regulación de caudales en un sistema tan fluctuante como el que nos ocupa tendrá efectos catastróficos tanto en el régimen hidráulico del río como en las comunidades aguas abajo.

La comparación de caudales circulantes dio como resultado cambios importantes tanto en la magnitud como en el régimen tras la construcción del embalse de El Pardo. Estos hechos se visualizan claramente en el gráfico de caudales medios mensuales a lo largo del periodo 1940-1994 (Fig. 1). Hasta el año 1970 se observa una cadencia regular que se repite anualmente y sin ninguna variación cualitativa de importancia: se produce un máximo $\left(9 \mathrm{~m}^{3} / \mathrm{s}\right)$ como consecuencia de las lluvias primaverales y, en el periodo de estiaje (julio a septiembre), el caudal se mantiene por debajo de $\operatorname{los} 2 \mathrm{~m}^{3} / \mathrm{s}$. A partir del año 1970 se rompe esta cadencia. Los caudales superiores a $4 \mathrm{~m}^{3} / \mathrm{s}$ son menos frecuentes, y los máximos anuales tienen un rango de oscilación mucho mayor (Fig. 3). Por otra parte, el máximo anual del periodo $\left(5.2 \mathrm{~m}^{3} / \mathrm{s}\right)$ se produce en el mes de marzo, es decir, además de reducirse en magnitud se ha adelantado en el tiempo (Fig. 2) Este desfase temporal tiene consecuencias dramáticas para la fauna piscícola autóctona, cuyas estrategias vitales están adaptadas a los ciclos hidrológicos naturales. Esta fuerte reducción en la magnitud de los caudales también se produce el resto del año. Así, el módulo natural $\left(4.48 \mathrm{~m}^{3} / \mathrm{s}\right)$ se reduce a menos de la mitad $\left(2.05 \mathrm{~m}^{3} / \mathrm{s}\right)$ tras la regulación (Fig. 2). Esto es debido, por una parte, al incremento de la capacidad del embalse de Santillana a partir del año 1969 que ha permitido destinar esta aportación "desaparecida" al consumo de Madrid mediante su conducción por el canal de Isabel II y, por otra a la construcción del embalse de El Pardo. 


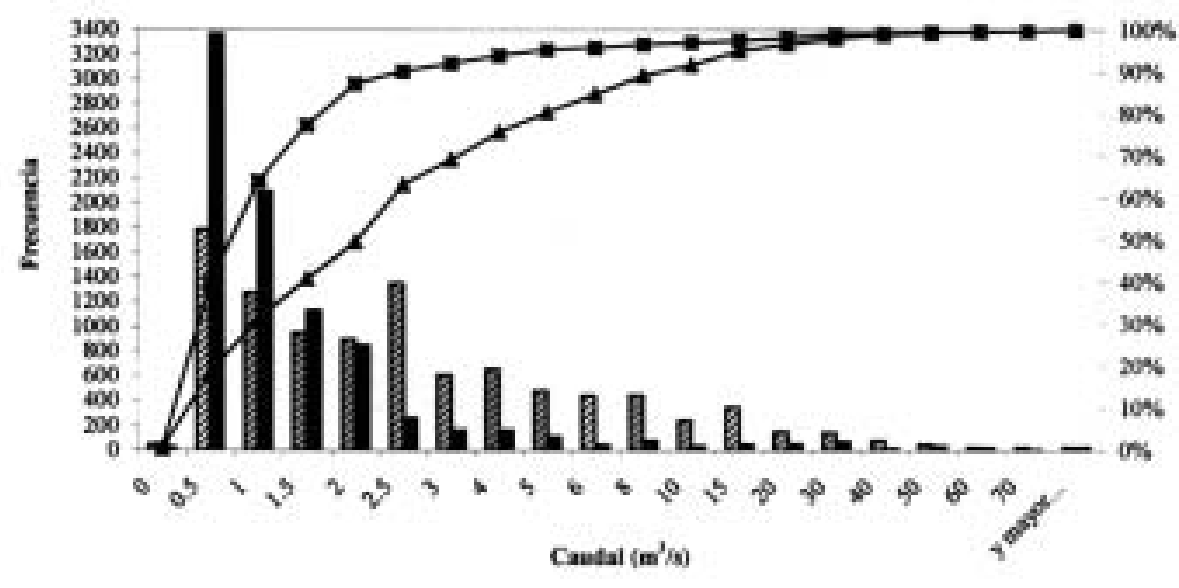

Eom Frecuencia Q4 42-70

Frocuencia Qd $70-94$

Facumalada Qd 42-70

Figura 3. Comparación de las frecuencias de caudales medios diarios antes y después de la construcción de la presa de El Pardo. Comparison of frequencies of daily average discharges before and after the construction of El Pardo reservoir.

Finalmente, se aprecia un máximo relativo en el mes de julio $\left(2.2 \mathrm{~m}^{3} / \mathrm{s}\right)$ provocado con objeto de limpiar el cauce y evitar los olores que se producen en el río Manzanares a su paso por Madrid en esta época del año (Fig. 2).

Este cambio de régimen de caudales ha repercutido en la morfología del tramo provocando una serie de cambios sintetizados en la tabla 5. En efecto, el trazado geomorfológico del cauce ha pasado de estar constituido por un cauce trenzado con gran tendencia a la sedimentación en forma de depósitos de arena (tipo D5 de Rosgen \& Lee, 1996), a un canal único, más estrecho y de mayor calado (tipo C), mejorando su estabilidad y capacidad de transporte de sedimentos. Como consecuencia, la vegetación ha colonizado orillas e isletas aumentando tanto la estabilidad del cauce como la capacidad de refugio para las poblaciones piscícolas leníticas.

\section{Las especies introducidas}

Por otra parte está el efecto de la introducción de especies exóticas, que se ve favorecido por el cambio del tipo de hábitat fluvial. En efecto, este tipo de acciones tendrá más probabilidades de éxito (en términos de la especie introducida) si las comunidades están gobernadas por factores abióticos, ya que la competencia por los recursos será mínima (Herbold \& Moyle, 1986), al igual que sucede en áreas de diversidad natural baja y, sobre todo, en áreas depauperadas, especialmente susceptibles a este tipo de acciones (Ross, 1991). El río Manzanares aguas abajo del embalse de El Pardo es un claro ejemplo. La fauna ictiológica del río Manzanares, bien conocida antes de sufrir las diversas actuaciones humanas (Velaz de Medrano \& Ugarte, 1933) estaba representada por la anguila (Anguilla anguilla) como depredador y numerosos ciprínidos como la boga de río (Chondrostoma polylepis), el cacho (Leuciscus cephalus), la tenca (Tinca tinca), dos especies de barbo (Barbus bocagei y Barbus comiza) y la lamprehuela (Cobitis paludica) (Tab. 6). Se trataba por tanto de una comunidad con una riqueza faunística relativamente baja, propia de los ríos mediterráneos.

Estudios posteriores (Doadrio, 1984) revelaron diversos cambios en la composición de la comunidad íctica. Así, aguas arriba del embalse de El Pardo, la comunidad íctica estaba compuesta tan solo por lucio, gobio y barbo común (Montes et al., 1987), mientras que aguas abajo 
Tabla 5. Características morfológicas del tramo antes y después de la construcción de la presa de El Pardo. Geomorphological features of the sampled stretch of River Manzanares.

\begin{tabular}{|c|c|c|c|c|c|c|c|c|c|}
\hline Especies & $\begin{array}{c}\text { Antes } \\
1930\end{array}$ & $\begin{array}{c}1930- \\
1939\end{array}$ & $\begin{array}{c}1930- \\
1949\end{array}$ & $\begin{array}{c}1950- \\
1960\end{array}$ & $\begin{array}{c}1960- \\
1970\end{array}$ & $\begin{array}{c}1970- \\
1982\end{array}$ & 1985 & 1987 & $\begin{array}{l}1989- \\
1997^{1}\end{array}$ \\
\hline $\begin{array}{l}\text { F. ANGUILLIDAE } \\
\text { A. anguilla }\end{array}$ & + & + & & & & & & & \\
\hline F. CYPRINIDAE & + & + & + & + & & + & & & + \\
\hline Ch. polylepis & + & + & & & + & + & & & \\
\hline L. cephalus & + & + & & + & & + & & & \\
\hline R. arcasii & + & + & + & + & + & + & + & + & + \\
\hline B. bocagei & + & & & & & & & & \\
\hline B. comiza & + & & & & & & & & \\
\hline T. tinca & & & & & & + & + & & + \\
\hline C. auratus & & & & & & + & + & + & + \\
\hline C. carpio & & & & & & + & + & + & + \\
\hline G. gobio & + & & & & & & & & \\
\hline \multicolumn{10}{|l|}{ F. COBITIDAE } \\
\hline C. paludica & + & + & & + & & + & + & + & \\
\hline \multicolumn{10}{|l|}{ F. ESOCIDAE } \\
\hline E. lucius & & & & + & & & + & & + \\
\hline \multicolumn{10}{|l|}{ F. POECILIIDAE } \\
\hline G. holbrooki & & & & & & & & + & + \\
\hline \multicolumn{10}{|c|}{ F. CENTRARCHIDAE } \\
\hline L. gibbosus & & & & & & & & + & + \\
\hline M. salmoides & & & & & & & & & + \\
\hline \multicolumn{10}{|l|}{ F. ICTALURIDAE } \\
\hline A. melas & & & & & & & & + & + \\
\hline \multicolumn{3}{|c|}{ Velaz de Medrano y Ugarte (1933) } & \multicolumn{3}{|c|}{ Casado et al (1987) } & & & & \\
\hline \multirow{2}{*}{\multicolumn{3}{|c|}{$\begin{array}{l}\text { Nieto }(1970) \\
\text { Doadrio }(1984)\end{array}$}} & \multicolumn{3}{|c|}{ Elvira (com. pers) } & & & & \\
\hline & & & ${ }^{1} \mathrm{Da}$ & os propi & & & & & \\
\hline \multicolumn{3}{|l|}{ Montes et al (1985) } & & & & & & & \\
\hline
\end{tabular}

la comunidad piscícola estaba compuesta por el barbo común como especie dominante, apareciendo como especies accesorias carpa (común y real), pez sol y pez gato (Casado et al., 1987, Cubillo et al., 1990).

En la actualidad tan solo permanecen la boga de río y el barbo común, lo que supone la desaparición de cuatro de las siete especies potenciales $(71.4 \%$ del total). Además, la aparición de la boga es básicamente puntual, no encontrándose una población mínimamente desarrollada. En cambio hemos encontrado numerosas especies exóticas de diversos orígenes: el lucio, probablemente procede del embalse, donde los Servicios de Caza y Pesca lo introdujeron como superdepredador al desaparecer la anguila por la construcción de presas en el río Tajo que impedían sus migraciones reproductivas al Océano Atlántico; el black-bass o perca americana, otro gran depredador exótico en nuestras aguas introducido en la Península por el Servicio de Caza y Pesca en el año 1955, para 
Tabla 6. Evolución de la comunidad piscícola del río Manzanares aguas abajo del embalse de El Pardo. Changes in the fish community in the River Manzanares downstream of El Pardo reservoir.

\begin{tabular}{|c|c|c|c|c|}
\hline Año & 1968 & 1972 & 1991 & $\%$ \\
\hline Anchura media (m) & 125.7 & - & 47.3 & -62.3 \\
\hline Superficie del cauce $\left(\mathrm{m}^{2}\right)$ & 79918.7 & 62517.9 & 56291.7 & -29.6 \\
\hline Superficie cubierta por veg. riparia $\left(\mathrm{m}^{2}\right)$ & 4624.5 & - & 37061.1 & +87.5 \\
\hline Tipo de río (Rosgen, 1996) & D5 & D5 & $\mathrm{C} 5$ & \\
\hline
\end{tabular}

pesca deportiva. Los ejemplares capturados en el área de estudio no son muy grandes pero sí se han observado individuos de gran tamaño aguas arriba (y que por lo tanto pueden acceder en épocas de crecidas al tramo estudiado).

La perca sol o pez sol, cuyo origen en este río probablemente se deba a la suelta incontrolada por parte de pescadores o a escapes (se usa como cebo vivo), es una especie, aunque de talla menor, de reconocida voracidad depredando sobre las puestas (observación personal) de otras especies lo que la convierte en un animal muy destructivo. Su potencial reproductivo es relativamente bajo (2000 huevos/hembra). No obstante, los datos de densidad obtenidos demuestran que tanto la perca sol como el black-bass se encuentra en clara expansión con los consiguientes efectos negativos sobre la comunidad íctica. Así, en muchas zonas la aclimatación de estas especies (pez sol, black-bass, lucio) produce serias alteraciones en nuestros ecosistemas acuáticos haciendo desaparecer algunas especies de la fauna local (Gómez, 1985). En concreto, en la cuenca del Tajo su extensión se ha visto fomentada recientemente al intentar hacer de esta especie una competidora seria del lucio (E. lucius) cuya expansión se intenta frenar (MOPT, 1991). En efecto, con frecuencia se han encontrado lucios muertos por las heridas producidas por los radios duros de las aletas dorsales de la perca sol tras su ingestión. No obstante, lejos de ser una medida correctora (dada su escasa incidencia) se está convirtiendo en un factor de propagación.

El pez gato es otra especie exótica, cuya presencia no es detectada hasta el año 1994. Sin embargo en un trabajo anterior (Casado et al.,
1987) ya se habían capturado algunos individuos de esta especie en el mismo tramo. Fue introducido en nuestro país a principios de siglo (1910) para pesca deportiva, y a finales de los años 80 ya se había extendido por las cuencas del Ebro, Ter y Tajo. Es una especie, que al igual que el resto de la familia, tolera bajas concentraciones de oxígeno y altos niveles de dióxido de carbono. Es por ello que suele encontrarse en el lecho del río, en áreas remansadas. Prefiere aguas cálidas y someras. Se alimenta a base de macroinvertebrados y huevos y larvas de otros peces (MOPT, 1991). Por otra parte, como hemos visto, la población de pez gato de este tramo del río Manzanares sufre constantes altibajos, no alcanzando nunca densidades elevadas que pudieran suponer tanto riesgo (por depredación, competencia por el espacio o el alimento) para el resto de la comunidad íctica como las especies anteriores. No obstante serían necesarios nuevos muestreos más específicos para confirmar este punto.

La gambusia, otra especie exótica, de origen norteamericano, fue introducida en el año 1955 como control biológico de la plaga del mosquito transmisor del paludismo. Hoy en día está ampliamente distribuida por toda la Península ya que se trata de una especie de amplio espectro ecológico, tolerante a gran variedad de condiciones desfavorables, y con un gran potencial reproductor.

Estos cambios observados en la comunidad íctica (desaparición de las especies autóctonas, sustituidas por especies exóticas, tras la construcción de un embalse) coinciden con los obtenidos por otros autores (Martínez et al., 1994) para el río Blanco (Colorado), donde la construcción de 
la presa Kennedy, la repoblación con especies exóticas (salmónidos sobre todo) y la introducción incontrolada de especies alóctonas por parte de los pescadores modificó totalmente la composición de la comunidad íctica que pasó de estar constituida en su totalidad por especies autóctonas tener a un $80 \%$ de especies exóticas (con $L e$ pomis cyanellus, L macrochirus y Micropterus salmoides entre otros). Efectos similares han sido referidos por Rodríguez et al. (1998) y Rodríguez-Ruiz (1998) para los ríos de la Península.

\section{CONCLUSIONES}

De la comunidad piscícola original tan sólo queda el barbo común, cuya población se encuentra envejecida, ya que de la boga de río únicamente podemos mencionar su presencia testimonial (Tabla 3). La comunidad está compuesta por un elevado porcentaje de especies alóctonas (80 $\%$ ), indicio de una comunidad degradada sometida a un fuerte estrés ambiental (regulación de caudales) que ha hecho que desaparezca la comunidad original dejando una serie de nichos vacíos que han sido ocupados por especies alóctonas introducidas por el hombre.

Por otra parte, las poblaciones ícticas son sumamente inestables y oscilan fuertemente a lo largo de los años (Tablas 2 a 4). Hay tres especies en expansión: el pez sol, el black-bass y el pez gato, todas ellas exóticas en nuestras aguas (Tabla 2).

El cambio de régimen ha sido un factor determinante en la evolución de la morfología y de la fauna piscícola. Tanto la menor frecuencia con la que ahora se producen las avenidas mayores de $4 \mathrm{~m}^{3} / \mathrm{s}$, como la mayor frecuencia de caudales bajos (entre 0.5 y $2.5 \mathrm{~m}^{3} / \mathrm{s}$ ), ha provocado una mejora en la estabilidad del tramo y una variación significativa en su morfología. El efecto directo del embalse mediante la regulación del caudal ha sido la transformación de un régimen de características torrenciales a otro mucho más homogéneo en cuanto a su rango de caudales extremos y con un carácter predominantemente lenítico. Como consecuencia la vegetación de ribera se ha establecido en las orillas proporcionando al biotopo de una mayor disponibilidad de refugio para los peces.

Este nuevo ecosistema ha sido propicio para el establecimiento de las especies introducidas en el río Manzanares como son la carpa (Cyprinus carpio), el pez sol (Lepomis gibbosus), el black-bass (Micropterus salmoides), el lucio (Esox lucius) y el pez gato (Ictalurus melas) todas ellas mejor adaptados a las condiciones de hábitat creadas por la regulación del embalse del El Pardo.

\section{BIBLIOGRAFÍA}

ANDERSON, R. O. \& R. M. NEUMAN. 1996. Length, weight, and associated structural indices. In: Fisheries Techniques. Brian R. Murphy \& David W. Willis (eds.) AFS, Bethesda, Maryland, USA.

CARLE, F. L. \& M. R. STRUB. 1978. A new method for estimating population size from removal data. Biometrics, 34: 321-380.

CASADO, C. \& D. GARCÍA DE JALÓN (coord.). 1987. Valoración ecológica de la red fluvial de la Comunidad de Madrid. Dir. Gral. de Recursos Hidraúlicos de la C.A.M.

CUBILlO, F., C. CASADO \& V. CASTILlO. 1990 Caudales ecológicos. Estudio de los regímenes de caudales mínimos en los cauces de la Comunidad de Madrid. De. Agencia de Medio Ambiente de la C.A.M.

DE VRIES, D. R. \& R.V. FRIE. 1996. Determination of Age and Growth. In: Fisheries Techniques. B. R. Murphy \& D. W. Willis (eds.) AFS, Bethesda, Maryland, USA.

DOADRIO, I. 1984. Relaciones filogenéticas y biogeográficas de los barbos (Barbus, Cyprinidae) de la Península Ibérica y aportes corológicos y biogeográficos a su ictiofauna continental. Tesis Doct. Univ. Complutense de Madrid.

DOADRIO, I., ELVIRA, B. \& BERNAT, Y. 1990. Peces continentales españoles. ICONA. $222 \mathrm{pp}$.

ELVIRA, B. 1996. Endangered freshwater fishes of Spain. In: Conservation of Endangered Freshwater Fishes in Europe. Kirchhofer \& Hefti (eds.). Birkhauser Verlag, Basel, Switzerland.

FAUSCH, K., J. LYONS, J. KARR \& P. ARGERMEIER. 1990. Fish communities as indicators or 
environmental degradation. Am. Fish. Soc. Symp., 8: $123-144$

GARCÍA DE JALÓN, D. 1987. River regulation in Spain. Reg. Rivers: Research. \& Managmt., 1: 343348.

GARCÍA DE JALÓN, D. 1990. Técnicas hidrobiológicas para la estimación de caudalesecológicos mínimos. In: Libro homenaje al Profesor D. M. García de Viedma. A. Ramos, A. Notario \& R. Baragaño (eds.): 183-196. FUCOVASA. UPM. Madrid.

GARCÍA DE JALÓN, D., M. GONZÁLEZ DEL TÁNAGO \& C. CASADO. 1992. Ecology of regulated streams in Spain: an overview. Limnetica, 8: 161-166

GARCÍA DE JALÓN, D., M. MAYO RUSTARAZO, F. HERVELLA, E. BARCELÓ \& T. FERNÁNDEZ. 1993. Principio y técnicas de gestión de la pesca en aguas continentales. Ed. Mundiprensa.

GARCÍA DE JALÓN, D., M. MAYO RUSTARAZO \& F. HERVELLA. 1996. Stock assessment and fisheries population dynamics in Spanish mountain streams. In: Stock Assessment in Inland Fisheries I.G. Cowx (ed.): 417-430. Fishing News Books, Blackwell Oxford.

GÓMEZ CARUANA, F. 1985. Especies exóticas de peces introducidas en los ríos. Quercus, 18: 17-18.

GRANADO-LORENCIO, C. 1992. Ecología de las especies piscícolas en los ecosistemas dulceacuícolas españoles. Limnetica, 8: 255-261.

GRANADO-LORENCIO, C. 1995. La ictiofauna de los ríos españoles: un patrimonio a conservar. Quercus, 111: 31-34.

GRANADO-LORENCIO, C. 1996. Ecología de peces. Univ. Sevilla.

HERBOLD, B \& P. B. MOYLE. 1986. Introduced species and vacant niches . Am. Naturalist., 128 (5): 751-760.

HORVÁTH, E. \& M. A. TOLEDO. 1998. Impacts of dams on fish fauna: feasibility of migration measures. In: 2 Int. PhD. Symposium in Civil Engineering.

LOBÓN-CERVIÁ, J. 1991. Dinámica de poblaciones de peces en ríos. Monografías del Museo Nacional de Ciencias Naturales-C.S.I.C. 156 pp.

LOBÓN-CERVIÁ, J. \& T. PENZACK. 1984. Fish production in the Jarama River, Central Spain. Hol. Ecol., 7: 128-137.

LOBÓN-CERVIÁ, J. \& B. ELVIRA. 1996. Estado de conservación de los peces fluviales ibéricos. Quercus, 44: 24-27.
MARTÍNEZ, P., T. CHART, M. TRAMMELL, WULLSSCHLEEGER, \& E. BWERGERSEN. 1994. Fish species composition before and after construction of a main stream reservoir on the White river, Colorado. Environm. Biol. Fish., 40: 227-239

MONTES, C., A. LLORCA \& A. STERLING. 1987. Directrices para la recuperación ecológica del tramo medio del río Manzanares. Ed. Canal de Isabel II. Madrid.142 pp.

MONTOYA, J. M. 1989. Pescar en Madrid. Agencia de Medio Ambiente de la C.A.M. 111pp.

M.O.P.T. 1991. Estudio de calidad de las aguas superficiales en cuanto a su aptitud para el baño y para la vida de los peces en la Cuenca del Tajo. Confederación Hidrográfica del Tajo.

MORÁN, P. A. P. 1951. A mathematical theory of animal trapping. Biometrica, 6: 250-258.

NIETO, M. 1970. Los pigmentos como indicadores ecológicos en las aguas corrientes del centro de España. C.E.I.A.A. Barcelona.

PECKARSKY, B. L. 1983. Biotic interactions or abiotic limitations? A model of lotic community structure. In: Dynamics of Lotic Ecosystems. T. D. Fontaine \& S. M. Bartell (eds.): 303-323. Ann Arbor Science, Ann Arbor, Michigan.

RODRIGUES, L. C., L. RIGADI, F. RIBEIRA, F. FILIPE, P. TIAGO, I COWX. \& M. J. COLLARES-PEREIRA. 1998. Os constrangimentos ambientais dos endemismos piscícolas ameaçados do troço português da bacia do Guadiana. I Congr. Iber. Limn., Évora, 1-6 Junio 1998.

RODRIGUEZ-RUIZ, A. 1998. Efecto de la construcción de un embalse sobre la ictiofauna en el tramo alto del río Guadalete (Sur Península Ibérica). I Congr. Iber. Limn., Évora, 1-6 Junio.

ROSGEN, D. L. \& LEE, H. 1996. Applied River Morphology. Wildland Hidrology, Pagosa Springs, Colorado.

ROSS, S. T. 1991. Mechanisms structuring stream fish assemblages: are there lessons from introduced species? Environm. Biol. Fishes, 30 (4): 359-368.

SERBER, G. A. F. \& E. D. L E CREN. 1967. Estimating populations parameters from catches large relative to the population. J. Anim. Ecol., 36: 631-643.

VANNOTE, R. L., G. W. MINSHALL, K.W. CUMMINS, J. R. SEDELL. \& C. E. CUSHING. 1980. The river continuum concept. Canad. J. Fish. Aquat. Sci., 37: 130-137.

VARELA, J. M. (coord.). 1996. Estudio de la vegetación de los ríos carpetanos de la cuenca del Jarama. Ed. CEDEX, Madrid. 
VELAZ DE MEDRANO, L. \& J. L. UGARTE. 1933. Estudio monográfico del río Manzanares. Ins. For. Invt. Exp., $\mathrm{n}^{\mathrm{o}}$ 1. Madrid. 68 pp.
WELCOMME, R. L. 1985. River fisheries. FAO Fisheries Technical Paper 262, 49 pp. 